To the Editors:

\title{
Measles, mumps, rubella (MMR) vaccine - a reply (2)
}

\author{
Ceylon Medical Journal 2011; 56: 186-187
}

The complications of mumps described in the recent letter published in the CMJ are well known [1]. Although the writer mentions that MMR has been given in developed countries for decades, the Japanese 2011 immunisation policy does not include mumps vaccine as mandatory - it is listed under the voluntary (optional) category which also includes hepatitis B, varicella, influenza, pneumococcus and Hib [2]. Only 2 out of 11 countries introduced mumps containing vaccines into their national immunisation schedules [3].

Although the MMR vaccine itself could cause hearing loss it is much less than the incidence of deafness due to the disease itself (approximately 5 in 100000 cases of mumps patients), which is mostly unilateral and often transient though permanent dysfunction may occur [4]. Therefore, most patients with deafness would not need cochlear implants as insinuated in the letter $[3,4,5]$. Some of the cases of deafness could not have been prevented even if immunisation was started after 2000, because some of those who developed deafness after mumps would have been older children and adults who would have missed the MMR anyway. Since nerve deafness has many other causes, attempts at costing morbidity following nonvaccination should be evidence based and not subjective impressions.

I was present at the 2000 Vaccination Summit, and voted for the measles/rubella vaccine. Considering the price of the vaccines the cost of having the MMR vaccine in 2002 would have been Rs. 70 million more than the MR vaccine [5]. There were other logistical issues that came up for consideration such as the size of the package and cold storage space.

The provocative insinuation "Those who opposed the MMR vaccine.... should bear responsibility for the occurrence of some cases of nerve deafness due to mumps" is an irresponsible statement by the author. Decisions regarding vaccination are made considering not only the need for the vaccine but also the cost. Initial priority would be to reduce mortality, and the measles vaccine was chosen initially mainly for that reason and because it was affordable with external UN funding. The measles vaccine was only introduced in the mid 1980's although the vaccine was available from the early sixties. Would the government, officials and medical professionals bear responsibility for the thousands of deaths caused by measles between the time when the measles vaccine was available and the time of its introduction in Sri Lanka; a span of more than 20 years?

The bivalent human papilloma virus (HPV) vaccine costs Rs. $3600 /=$ per dose and if $50 \%$ of annual births (about 175,000 girls) are given the vaccine (3 doses) the cost would be Rs. 1890, 000,000/= per year. Can we afford this in Sri Lanka although reasons for its introduction are logical? Use of provocative statements are dangerous since women who may develop cervical cancer later in their lives would accuse those responsible for making decisions regarding its inclusion or exclusion from the EPI.

We may be offered expensive vaccines at concessionary rates or free for a limited period of time by the GAVI foundation or UN agencies. Once we include it into the EPI we cannot withdraw it when the funding ceases; we then have to buy it. Although there is an argument that vaccine prices are likely to fall, this often does not happen. Foreign organisations may introduce such vaccine/s to many other countries and demand would increase, and depending on supply, the price may not come down. For example the price of 10 dose MMR in 2004 was $\$ 1.150$ per dose while the 2010 price was US\$1.6, and the price of 10 dose MR was US\$ 0.48 per dose in 2002 while the 2010 price was US\$ 0.4840 [6]. We should not take on new vaccines just because somebody is paying for it in the initial few years. There has to be evidence based need for the vaccine, and the cost to the state should also be considered before taking decisions. Now that we can afford the MMR, we should give it without blaming previous majority decisions which were probably rational at the time they were made.

\section{References}

1. Lamabadusuriya SP. Measles, mumps, rubella (MMR) vaccine. Ceylon Medical Journal 2011; 56: 135.

2. Immunization schedule Japan 2011 for those under 20 years of age http://idsc.nih.go.jp/vaccine/dschedule/Imm11EN.pdf

3. Introduction of mumps measles rubella vaccine into the National Immunization Programme. Epidemiological Unit, Ministry of Health 2011. http://www.epid.gov.lk/pdf/ Immunization/MMR\%20Introduction\%20.pdf

4. Deafness following mumps: the possible pathogenesis and incidence of deafness.Virology-Online. http://virologyonline.com/viruses/MUMPS.htm 
5. Mizushima N, Murakami Y. Immunization science measles, mumps vaccination and hearing loss. Auris Nasus Larynx 1986; 13: S55-7.

http://www.ncbi.nlm.nih.gov/pubmed/3767776http:// www.immunizationinfo.org/science/measles-mumpsvaccination-and-hearing-loss

6. http://www.unicef.org/supply/index_57476.html

\section{G H de Silva}

Department of Paediatrics, University of Kelaniya, Sri Lanka.

This Correspondence is closed.

\section{Joint Editors, CMJ}

